\title{
Consistency of Hill Estimators in a Linear Preferential Attachment Model
}

\author{
Tiandong Wang · Sidney I. Resnick
}

Received: November 15, 2017

\begin{abstract}
Preferential attachment is widely used to model power-law behavior of degree distributions in both directed and undirected networks. Practical analyses on the tail exponent of the power-law degree distribution use Hill estimator as one of the key summary statistics, whose consistency is justified mostly for iid data. The major goal in this paper is to answer the question whether the Hill estimator is still consistent when applied to non-iid network data. To do this, we first derive the asymptotic behavior of the degree sequence via embedding the degree growth of a fixed node into a birth immigration process. We also need to show the convergence of the tail empirical measure, from which the consistency of Hill estimators is obtained. This step requires checking the concentration of degree counts. We give a proof for a particular linear preferential attachment model and use simulation results as an illustration in other choices of models.
\end{abstract}

Keywords Hill estimators · Power laws · Preferential Attachment · Continuous Time Branching Processes

Mathematics Subject Classification (2010) 60G70 - 60B10 - 60G55 . $60 \mathrm{G} 57 \cdot 05 \mathrm{C} 80 \cdot 62 \mathrm{E} 20$

This work was supported by Army MURI grant W911NF-12-1-0385 to Cornell University.

Tiandong Wang

School of Operations Research and Information Engineering, Cornell University, Ithaca, NY 14853

E-mail: tw398@cornel.edu

Sidney I. Resnick

School of Operations Research and Information Engineering, Cornell University, Ithaca, NY 14853

E-mail: sir1@cornel.edu 


\section{Introduction.}

The preferential attachment model gives a random graph in which nodes and edges are added to the network based on probabilistic rules, and is used to mimic the evolution of networks such as social networks, collaborator and citation networks, as well as recommender networks. The probabilistic rule depends on the node degree and captures the feature that nodes with larger degrees tend to attract more edges. Empirical analysis of social network data shows that degree distributions follow power laws. Theoretically, this is true for linear preferential attachment models which makes preferential attachment a popular choice for network modeling (Bollobás et al., 2003, Durrett, 2010 Krapivsky et al., 2001, Krapivsky and Redner, 2001: van der Hofstad, 2017). The preferential attachment mechanism has been applied to both directed and undirected graphs. Limit theory for degree counts can be found in Resnick and Samorodnitsky (2016), Bhamidi (2007), Krapivsky and Redner (2001) for the undirected case and Wang and Resnick (2017), Samorodnitsky et al. (2016), Resnick and Samorodnitsky (2015), Wang and Resnick (2016), Krapivsky et al. (2001) for the directed case. This paper only focuses on the undirected case.

One statistical issue is how to estimate the index of the degree distribution power-law tail. In practice, this is often done by combining a minimum distance method Clauset et al. (2009) with the Hill estimator Hill (1975). Data repositories of large network datasets such as KONECT (http://konect.unikoblenz.de/) Kunegis (2013) provide for each dataset key summary statistics including Hill estimates of degree distribution tail indices. However, there is no theoretical justification for such estimates and consistency of the Hill estimator has been proved only for data from a stationary sequence of random variables, which is assumed to be either iid Mason (1982) or satisfy certain structural or mixing assumptions, e.g. Hsing (1991); Resnick and Stărică (1995, 1998); Rootzén et al. (1990). Therefore, proving/disproving the consistency of Hill estimators for network data is a major concern in this paper.

The Hill estimator and other tail descriptors are often analyzed using the tail empirical estimator. Using standard point measure notation, let

$$
\epsilon_{x}(A)=\left\{\begin{array}{ll}
1, & \text { if } x \in A \\
0, & \text { if } x \notin A
\end{array} .\right.
$$

For positive iid random variables $\left\{X_{i}: i \geq 1\right\}$ whose distribution has a regularly varying tail with index $-\alpha<0$, we have the following convergence in the space of Radon measures on $(0, \infty]$ of the sequence of empirical measures

$$
\sum_{i=1}^{n} \epsilon_{X_{i} / b(n)}(\cdot) \Rightarrow \operatorname{PRM}\left(\nu_{\alpha}(\cdot)\right), \quad \text { with } \quad \nu_{\alpha}(y, \infty]=y^{-\alpha}, y>0,
$$

to the limit Poisson random measure with mean measure $\nu_{\alpha}(\cdot)$. From 1.1 other extremal properties of $\left\{X_{n}\right\}$ follow (Resnick, 1987, Chapter 4.4). See for example the application given in this paper after Theorem 5 . Further, for any 
intermediate sequence $k_{n} \rightarrow \infty, k_{n} / n \rightarrow 0$ as $n \rightarrow \infty$, the sequence of tail empirical measures also converge to a deterministic limit,

$$
\frac{1}{k_{n}} \sum_{i=1}^{n} \epsilon_{X_{i} / b\left(n / k_{n}\right)}(\cdot) \Rightarrow \nu_{\alpha}(\cdot),
$$

which is one way to prove consistency of the Hill estimator for iid data Resnick (2007. Chapter 4.4). We seek a similar dual pair as (1.1) and 1.2 for network models that facilitates the study of the Hill estimator and extremal properties of node degrees.

With this goal in mind, we first find the limiting distribution for the degree sequence in a linear preferential attachment model, from which a similar convergence result to (1.1) follows. Embedding the network growth model into a continuous time branching process (cf. Athreya (2007); Athreya et al. (2008); Bhamidi (2007)) is a useful tool in this case. We model the growth of the degree of each single node as a birth process with immigration. Whenever a new node is added to the network, a new birth immigration process is initiated. In this embedding, the total number of nodes in the network growth model also forms a birth immigration process. Using results from the limit theory of continuous time branching processes (cf. Resnick (1992, Chapter 5.11); Tavaré (1987)), we give the limiting distribution of the degree of a fixed node as well as the maximal degree growth.

Empirical evidence for simulated networks leads to the belief that the Hill estimator is consistent. However, proving the analogue of 1.2 is challenging and requires showing concentration inequalities for expected degree counts. We have only succeeded for a particular linear preferential attachment model, where each new node must attach to one of the existing nodes in the graph. We are not sure the concentration inequalities always hold for preferential attachment and discussion of limitations of the Hill estimator for network data must be left for the future. For a more sophisticated model where we could not verify the concentration inequalities, we illustrate consistency of the Hill estimator coupled with a minimum distance method (introduced in Clauset et al. (2009) ) via simulation for a range of parameter values; however the asymptotic distribution of the Hill estimator in this case is confounding and it is not obviously normal. Whether this possible non-normality is due to the minimum distance threshold selection or due to network data (rather than iid data) being used, we are not sure at this point.

The rest of the paper is structured as follows. After giving background on the tail empirical measure and Hill estimator in the rest of this section, Section 2 gives two linear preferential attachment models. Section 3 summarizes key facts about the pure birth and the birth-immigration processes. We analyze social network degree growth in Section 4 using a sequence of birthimmigration processes and give the limiting empirical measures of normalized degrees in the style of (1.1) for both models under consideration. We prove the consistency of the Hill estimator for the simpler model in Section 5 and give simulation results in Section 6 that illustrate the behavior of Hill estimators in the other model. 
Parameter estimation based on maximum likelihood or approximate MLE for directed preferential attachment models is studied in Wan et al. (2017). A comparison between MLE model based methods and asymptotic extreme value methods is forthcoming.

\subsection{Background}

Our approach to the Hill estimator considers it as a functional of the tail empirical measure so we start with necessary background and review standard results (cf. Resnick (2007, Chapter 3.3.5)).

For $\mathbb{E}=(0, \infty]$, let $M_{+}(\mathbb{E})$ be the set of non-negative Radon measures on $\mathbb{E}$. A point measure $m$ is an element of $M_{+}(\mathbb{E})$ of the form

$$
m=\sum_{i} \epsilon_{x_{i}}
$$

The set $M_{p}(\mathbb{E})$ is the set of all Radon point measures of the form $(1.3)$ and $M_{p}(\mathbb{E})$ is a closed subset of $M_{+}(\mathbb{E})$ in the vague metric.

For $\left\{X_{n}, n \geq 1\right\}$ iid and non-negative with common regularly varying distribution tail $\bar{F} \in R V_{-\alpha}, \alpha>0$, there exists a sequence $\{b(n)\}$ such that for a limiting Poisson random measure with mean measure $\nu_{\alpha}$ and $\nu_{\alpha}(y, \infty]=y^{-\alpha}$ for $y>0$, written as $\operatorname{PRM}\left(\nu_{\alpha}\right)$, we have

$$
\sum_{i=1}^{n} \epsilon_{X_{i} / b(n)} \Rightarrow \operatorname{PRM}\left(\nu_{\alpha}\right), \quad \text { in } M_{p}((0, \infty]),
$$

and for some $k_{n} \rightarrow \infty, k_{n} / n \rightarrow 0$,

$$
\frac{1}{k_{n}} \sum_{i=1}^{n} \epsilon_{X_{i} / b\left(n / k_{n}\right)} \Rightarrow \nu_{\alpha}, \quad \text { in } M_{+}((0, \infty]),
$$

Note the limit in 1.4 is random while that in 1.5 is deterministic. Define the Hill estimator $H_{k, n}$ based on $k$ upper order statistics of $\left\{X_{1}, \ldots, X_{n}\right\}$ as in Hill (1975)

$$
H_{k, n}:=\frac{1}{k} \sum_{i=1}^{k} \log \frac{X_{(i)}}{X_{(k+1)}},
$$

where $X_{(1)} \geq X_{(2)} \geq \ldots \geq X_{(n)}$ are order statistics of $\left\{X_{i}: 1 \leq i \leq n\right\}$. In the iid case there are many proofs of consistency (cf. Csörgö et al. (1991a); de Haan and Resnick (1998); Hall (1982); Mason (1982); Mason and Turova (1994)): For $k=k_{n} \rightarrow \infty, k_{n} / n \rightarrow 0$, we have

$$
H_{k_{n}, n} \stackrel{P}{\rightarrow} 1 / \alpha \quad \text { as } n \rightarrow \infty .
$$

The treatment in Resnick (2007, Theorem 4.2) approaches consistency by showing 1.6 follows from (1.5) and we follow this approach for the network context where the iid case is inapplicable. The next section constructs two 
undirected preferential attachment models, labelled A and B, and gives behavior of $D_{i}(n)$, the degree of node $i$ at the $n$th stage of construction. Theorem 5 shows that for $\delta$, a parameter in the model construction, the degree sequences in either Model A or B have empirical measures

$$
\sum_{i=1}^{n} \epsilon_{D_{i}(n) / n^{1 /(2+\delta)}}
$$

that converge weakly to some random limit point measure in $M_{p}((0, \infty])$. The question then becomes whether there is an analogy to 1.5 in the network case so that

$$
\frac{1}{k_{n}} \sum_{i=1}^{n} \epsilon_{D_{i}(n) / b\left(n / k_{n}\right)} \Rightarrow \nu_{2+\delta}, \quad \text { in } M_{+}((0, \infty]),
$$

with some function $b(\cdot)$ and intermediate sequence $k_{n}$. This would facilitate proving consistency of the Hill estimator. We successfully derive (1.8) for Model A in Section 5 and discuss why we failed for Model B. For Model A, we give the consistency of the Hill estimator.

\section{Preferential Attachment Models.}

\subsection{Model setup.}

We consider an undirected preferential attachment model initiated from the initial graph $G(1)$, which consists of one node $v_{1}$ and a self loop. Node $v_{1}$ then has degree 2 at stage $n=1$. For $n \geq 1$, we obtain a new graph $G(n+1)$ by appending a new node $v_{n+1}$ to the existing graph $G(n)$. The graph $G(n)$ consists of $n$ edges and $n$ nodes. Denote the set of nodes in $G(n)$ by $V(n):=$ $\left\{v_{1}, v_{2}, \ldots, v_{n}\right\}$. For $v_{i} \in V(n), D_{i}(n)$ is the degree of $v_{i}$ in $G(n)$. We consider two ways to construct the random graph and refer to them as Model A and B.

Model A: Given $G(n)$, the new node $v_{n+1}$ is connected to one of the existing nodes $v_{i} \in V(n)$ with probability

$$
\frac{f\left(D_{i}(n)\right)+\delta}{\sum_{i=1}^{n}\left(f\left(D_{i}(n)\right)+\delta\right)}
$$

where the preferential attachment function $f(j), j \geq 1$ is deterministic and non-decreasing. In this case, the new node $v_{n+1}$ for $n \geq 1$, is always born with degree 1.

Model B: In this model, given graph $G(n)$, the graph $G(n+1)$ is obtained by either: 
- Adding a new node $v_{n+1}$ and a new edge connecting to an existing node $v_{i} \in V(n)$ with probability

$$
\frac{f\left(D_{i}(n)\right)+\delta}{\sum_{i=1}^{n}\left(f\left(D_{i}(n)\right)+\delta\right)+f(1)+\delta},
$$

where $\delta>-f(1)$ is a parameter;

or

- Adding a new node $v_{n+1}$ with a self loop with probability

$$
\frac{f(1)+\delta}{\sum_{i=1}^{n}\left(f\left(D_{i}(n)\right)+\delta\right)+f(1)+\delta} .
$$

Linear case: If the preferential attachment function is $f(j)=j$ for $j=$ $1,2, \ldots$, then the model is called the linear preferential attachment model. Since every time we add a node and an edge the degree of 2 nodes is increased by 1 , we have for both model $\mathrm{A}$ or $\mathrm{B}$ that $\sum_{i=1}^{n} D_{i}(n)=2 n, n \geq 1$. Therefore, the attachment probabilities in (2.1), 2.2) and (2.3) are

$$
\frac{D_{i}(n)+\delta}{(2+\delta) n}, \quad \frac{D_{i}(n)+\delta}{(2+\delta) n+1+\delta}, \quad \text { and } \quad \frac{1+\delta}{(2+\delta) n+1+\delta},
$$

respectively, where $\delta>-1$ is a constant.

\subsection{Power-law tails.}

Continuing with $f(j)=j$, suppose $G(n)$ is a random graph generated by either Model A or B after $n$ steps. Let $N_{k}(n)$ be the number of nodes in $G(n)$ with degree equal to $k$, i.e.

$$
N_{k}(n):=\sum_{i=1}^{n} \mathbf{1}_{\left\{D_{i}(n)=k\right\}},
$$

then $N_{>k}(n):=\sum_{j>k} N_{j}(n), k \geq 1$, is the number of nodes in $G(n)$ with degree strictly greater than $k$. For $k=0$, we set $N_{>0}(n)=n$.

For both models A and B, it is shown in van der Hofstad (2017, Theorem 8.3) using concentration inequalities and martingale methods that for fixed $k \geq 1$, as $n \rightarrow \infty$,

$$
\frac{N_{k}(n)}{n} \stackrel{P}{\rightarrow} p_{k}=(2+\delta) \frac{\Gamma(k+\delta) \Gamma(3+2 \delta)}{\Gamma(k+3+2 \delta) \Gamma(1+\delta)} \sim(2+\delta) \frac{\Gamma(3+2 \delta)}{\Gamma(1+\delta)} k^{-(3+\delta)} ;
$$

$\left(p_{k}\right)_{k \geq 0}$ is a pmf and the asymptotic form, as $k \rightarrow \infty$, follows from Stirling. Let $p_{>k}=\sum_{i>k} p_{j}$ be the complementary cdf and by Scheffé's lemma as well as van der Hofstad (2017, Equation (8.4.6)), we have

$$
\frac{N_{>k}(n)}{n} \stackrel{P}{\rightarrow} p_{>k}:=\frac{\Gamma(k+1+\delta) \Gamma(3+2 \delta)}{\Gamma(k+3+2 \delta) \Gamma(1+\delta)},
$$


and again by Stirling's formula we get from (2.6) as $k \rightarrow \infty$,

$$
p_{>k} \sim c \cdot k^{-(2+\delta)}, \quad c=\frac{\Gamma(3+2 \delta)}{\Gamma(1+\delta)} .
$$

In other words, the tail distribution of the asymptotic degree sequence in a linear preferential attachment model is asymptotic to a power law with tail index $2+\delta$.

In practice, the Hill estimator is widely used to estimate this tail index. Absent prior justification for using the Hill estimator on network data, we investigate its use.

\section{Preliminaries: Continuous Time Markov Branching Processes.}

In this section, we review two continuous time Markov branching processes needed in Section 4.1, where we embed the degree sequence of a fixed network node into a continuous time branching process and derive the asymptotic limit of the degree growth.

\subsection{Linear birth processes.}

A linear birth process $\{\zeta(t): t \geq 0\}$ is a continuous time Markov process taking values in the set $\mathbb{N}^{+}=\{1,2,3, \ldots\}$ and having a transition rate

$$
q_{i, i+1}=\lambda i, \quad i \in \mathbb{N}^{+}, \quad \lambda>0 .
$$

The linear birth process $\{\zeta(t): t \geq 0\}$ is a mixed Poisson process; see Resnick (1992, Theorem 5.11.4), Kendall (1966) and Waugh (1970) among other sources. If $\zeta(0)=1$ then the representation is

$$
\zeta(t)=1+N_{0}\left(W\left(e^{\lambda t}-1\right), t \geq 0\right.
$$

where $\left\{N_{0}(t): t \geq 0\right\}$ is a unit rate homogeneous Poisson on $\mathbb{R}_{+}$with $N_{0}(0)=$ 0 and $W \Perp N_{0}(\cdot)$ is a unit exponential random variable independent of $N_{0}$. Since $N_{0}(t) / t \rightarrow 1$ almost surely as $t \rightarrow \infty$, it follows immediately that

$$
\frac{\zeta(t)}{e^{\lambda t}} \stackrel{\text { a.s. }}{\longrightarrow} W, \quad \text { as } t \rightarrow \infty
$$

We use these facts in Section 4.2 to analyze the asymptotic behavior of the degree growth in a preferential attachment network. 
3.2 Birth processes with immigration.

Apart from individuals within the population giving birth to new individuals, population size can also increase due to immigration which is assumed independent of births. The linear birth process with immigration (B.I. process), $\{B I(t): t \geq 0\}$, having lifetime parameter $\lambda>0$ and immigration parameter $\theta \geq 0$ is a continuous time Markov process with state space $\mathbb{N}=\{0,1,2,3, \ldots\}$ and transition rate

$$
q_{i, i+1}=\lambda i+\theta .
$$

When $\theta=0$ there is no immigration and the B.I. process becomes a pure birth process.

For $\theta>0$, the B.I. process starting from 0 can be constructed from a Poisson process and an independent family of iid linear birth processes Tavaré (1987). Suppose that $N_{\theta}(t)$ is the counting function of homogeneous Poisson points $0<\tau_{1}<\tau_{2}<\ldots$ with rate $\theta$ and independent of this Poisson process we have independent copies of a linear birth process $\left\{\zeta_{i}(t): t \geq 0\right\}_{i \geq 1}$ with parameter $\lambda>0$ and $\zeta_{i}(0)=1$ for $i \geq 1$. Let $B I(0)=0$, then the B.I. process is a shot noise process with form

$$
B I(t):=\sum_{i=1}^{\infty} \zeta_{i}\left(t-\tau_{i}\right) \mathbf{1}_{\left\{t \geq \tau_{i}\right\}}=\sum_{i=1}^{N_{\theta}(t)} \zeta_{i}\left(t-\tau_{i}\right) .
$$

Theorem 1 modifies slightly the statement of Tavaré (1987, Theorem 5) summarizing the asymptotic behavior of the B.I. process.

Theorem 1 For $\{B I(t): t \geq 0\}$ as in (3.3), we have as $t \rightarrow \infty$,

$$
e^{-\lambda t} B I(t) \stackrel{a . s s}{\longrightarrow} \sum_{i=1}^{\infty} W_{i} e^{-\lambda \tau_{i}}=: \sigma
$$

where $\left\{W_{i}: i \geq 1\right\}$ are independent unit exponential random variables satisfying for each $i \geq 1$,

$$
W_{i}=\lim _{t \rightarrow \infty} e^{-t} \zeta_{i}(t) .
$$

The random variable $\sigma$ in (3.4) is a.s. finite and has a Gamma density given by

$$
f(x)=\frac{1}{\Gamma(\theta / \lambda)} x^{\theta / \lambda-1} e^{-x}, \quad x>0 .
$$

The form of $\sigma$ in (3.4) and its Gamma density is justified in Tavaré (1987). It can be guessed from (3.3) and some cavalier interchange of limits and infinite sums. The density of $\sigma$ comes from transforming Poisson points $\left\{\left(W_{i}, \tau_{i}\right), i \geq\right.$ $1\}$, summing and recognizing a Gamma Lévy process at $t=1$. 


\section{Embedding Process.}

Our approach to the weak convergence of the sequence of empirical measures in 1.7) embeds the degree sequences $\left\{D_{i}(n), 1 \leq i \leq n, n \geq 1\right\}$ into a B.I. process. The embedding idea is proposed in Athreya et al. (2008) and we tailor it for our setup finding it flexible enough to accommodate both linear preferential attachment Models A and B introduced in Section 2.1.

\subsection{Embedding.}

Here is how we embed the network growth model using a sequence of independent B.I. processes.

\subsubsection{Model $A$ and B.I. processes.}

Model A is the simpler case where a new node is not allowed to have self loop. Let $\left\{B I_{i}(t): t \geq 0\right\}_{i \geq 1}$ be independent B.I. processes such that

$$
B I_{1}(0)=2, \quad B I_{i}(0)=1, \quad \forall i \geq 2 .
$$

Each has transition rate is $q_{j, j+1}=j+\delta, \delta>-1$. For $i \geq 1$, let $\left\{\tau_{k}^{(i)}: k \geq 1\right\}$ be the jump times of the B.I. process $\left\{B I_{i}(t): t \geq 0\right\}$ and set $\tau_{0}^{(i)}:=0$ for all $i \geq 1$. Then for $k \geq 1$,

$$
B I_{1}\left(\tau_{k}^{(1)}\right)=k+2, \quad B I_{i}\left(\tau_{k}^{(i)}\right)=k+1, i \geq 2 .
$$

Therefore,

$$
\tau_{k}^{(1)}-\tau_{k-1}^{(1)} \sim \operatorname{Exp}(k+1+\delta), \quad \text { and } \quad \tau_{k}^{(i)}-\tau_{k-1}^{(i)} \sim \operatorname{Exp}(k+\delta), i \geq 2 .
$$

and $\left\{\tau_{k}^{(i)}-\tau_{k-1}^{(i)}: i \geq 1, k \geq 1\right\}$ are independent.

Set $T_{1}^{A}=0$ and relative to $B I_{1}(\cdot)$ define

$$
T_{2}^{A}:=\tau_{1}^{(1)},
$$

i.e. the first time that $B I_{1}(\cdot)$ jumps. Start the new B.I. process $\left\{B I_{2}\left(t-T_{2}^{A}\right)\right.$ : $\left.t \geq T_{2}^{A}\right\}$ at $T_{2}^{A}$ and let $T_{3}^{A}$ be the first time after $T_{2}^{A}$ that either $B I_{1}(\cdot)$ or $B I_{2},(\cdot)$ jumps so that,

$$
T_{3}^{A}=\min \left\{T_{i}^{A}+\tau_{k}^{(i)}: k \geq 1, T_{i}^{A}+\tau_{k}^{(i)}>T_{2}^{A}, i=1,2\right\} .
$$

Start a new, independent B.I. process $\left\{B I_{3}\left(t-T_{3}^{A}\right)\right\}_{t \geq T_{3}^{A}}$ at $T_{3}^{A}$. See Figure 4.1 which assumes $\tau_{1}^{(2)}+T_{2}^{A}<\tau_{2}^{(1)}$. Continue in this way. When $n$ lines have been created, define $T_{n+1}^{A}$ to be the first time after $T_{n}^{A}$ that one of the processes $\left\{B I_{i}\left(t-T_{i}^{A}\right): t \geq T_{i}^{A}\right\}_{1 \leq i \leq n}$ jumps, i.e.

$$
T_{n+1}^{A}:=\min \left\{T_{i}^{A}+\tau_{k}^{(i)}: k \geq 1, T_{i}^{A}+\tau_{k}^{(i)}>T_{n}^{A}, 1 \leq i \leq n\right\} .
$$

At $T_{n+1}^{A}$, start a new, independent B.I. process $\left\{B I_{n+1}\left(t-T_{n+1}^{A}\right)\right\}_{t \geq T_{n+1}^{A}}$. 


$$
\begin{aligned}
& B I_{1}(0)=2 \quad B I_{1}\left(T_{2}^{A}\right)=3
\end{aligned}
$$

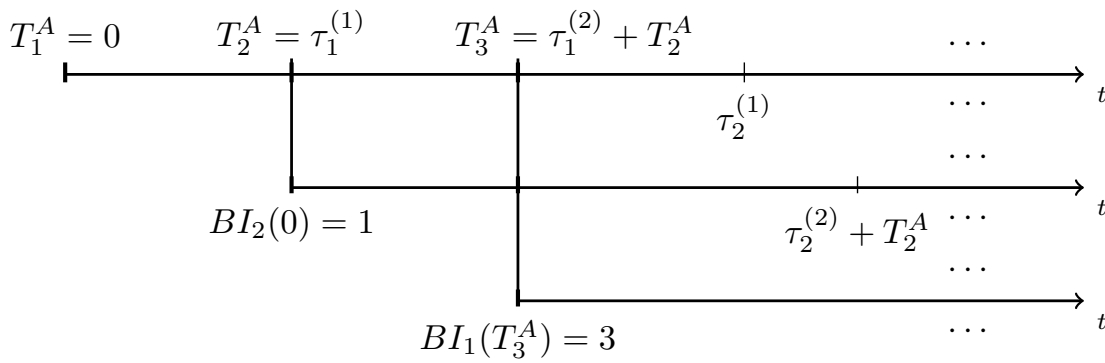

$$
\begin{aligned}
& B I_{2}\left(T_{3}^{A}-T_{2}^{A}\right)=2 \\
& B I_{3}(0)=1
\end{aligned}
$$

Fig. 4.1 Embedding procedure for Model A assuming $\tau_{1}^{(2)}+T_{2}^{A}<\tau_{2}^{(1)}$.

\subsubsection{Model B and BI processes.}

In Model B, a new node may be born with a self loop but the B.I. process framework can still be used. We keep the independent sequence of $\left\{B I_{i}(t)\right.$ : $t \geq 0\}_{i \geq 1}$ initialized as in 4.1, as well as the definition of $\left\{\tau_{k}^{(i)}: k \geq 1\right\}$ for $i \geq 1$.

Set $T_{0}^{B}=T_{1}^{B}=0$ and start two B.I. processes $B I_{1}(\cdot)$ and $B I_{2}(\cdot)$ at $T_{1}^{B}$. At time $T_{n}^{B}$ with $n \geq 1$, there exist $n+1$ B.I. processes. We define $T_{n+1}^{B}$ as the first time after $T_{n}^{B}$ that one of the processes $\left\{B I_{i}\left(t-T_{i-1}^{B}\right): t \geq T_{i-1}^{B}\right\}_{1 \leq i \leq n+1}$ jumps, i.e.

$$
T_{n+1}^{B}:=\min \left\{T_{i-1}^{B}+\tau_{k}^{(i)}: k \geq 1, T_{i-1}^{B}+\tau_{k}^{(i)}>T_{n}^{B}, 1 \leq i \leq n+1\right\},
$$

and start a new, independent B.I. process $\left\{B I_{n+2}\left(t-T_{n+1}^{B}\right)\right\}_{t \geq T_{n+1}^{B}}$ at $T_{n+1}^{B}$.

\subsubsection{Embedding.}

The following embedding theorem is similar to the one proved in Athreya et al. (2008) and summarizes how to embed in the B.I. constructions.

Theorem 2 Fix $n \geq 1$.

(a) For Model A, suppose

$$
\mathcal{D}^{A}(n):=\left(D_{1}^{A}(n), \ldots, D_{n}^{A}(n)\right)
$$

is the degree sequence of nodes in the graph $G(n)$ and $\left\{T_{n}^{A}\right\}_{n \geq 1}$ is defined as in 4.3. For each fixed $n$, define

$$
\widetilde{\mathcal{D}}^{A}(n):=\left(B I_{1}\left(T_{n}^{A}\right), B I_{2}\left(T_{n}^{A}-T_{2}^{A}\right), \ldots, B I_{n-1}\left(T_{n}^{A}-T_{n-1}^{A}\right), B I_{n}(0)\right),
$$


and then $\mathcal{D}^{A}(n)$ and $\widetilde{\mathcal{D}}^{A}(n)$ have the same distribution in $\mathbb{R}^{n}$.

(b) Analogously, for Model B, the degree sequence

$$
\mathcal{D}^{B}(n):=\left(D_{1}^{B}(n), \ldots, D_{n}^{B}(n)\right)
$$

and

$$
\widetilde{\mathcal{D}}^{B}(n):=\left(B I_{1}\left(T_{n}^{B}\right), B I_{2}\left(T_{n}^{B}\right), \ldots, B I_{n-1}\left(T_{n}^{B}-T_{n-2}^{B}\right), B I_{n}\left(T_{n}^{B}-T_{n-1}^{B}\right)\right)
$$

have the same distribution in $\mathbb{R}^{n}$.

Proof By the construction of Model A, at each $T_{n}^{A}, n \geq 2$, we start a new B.I. process $B I_{n}(\cdot)$ with initial value equal to 1 and one of $B I_{i}, 1 \leq i \leq n-1$ also increases by 1 . This makes the sum of the values of $B I_{i}, 1 \leq i \leq n$, increase by 2 so that

$$
\sum_{i=1}^{n}\left(B I_{i}\left(T_{n}^{A}-T_{i}^{A}\right)+\delta\right)=(2+\delta) n
$$

The rest is essentially the proof of Athreya et al. (2008, Theorem 2.1) which we now outline.

Both $\left\{\mathcal{D}^{A}(n), n \geq 1\right\}$ and $\left\{\widetilde{\mathcal{D}}^{A}(n), n \geq 1\right\}$ are Markov on the state space $\cup_{n \geq 1} \mathbb{R}_{+}^{n}$ since

$$
\begin{aligned}
& \mathcal{D}^{A}(n+1)=\left(\mathcal{D}^{A}(n), 1\right)+\left(\boldsymbol{e}_{J_{n+1}}^{(n)}, 0\right), \\
& \widetilde{\mathcal{D}}^{A}(n+1)=\left(\widetilde{\mathcal{D}}^{A}(n), 1\right)+\left(\boldsymbol{e}_{L_{n+1}}^{(n)}, 0\right),
\end{aligned}
$$

where for $n \geq 1, \boldsymbol{e}_{j}^{(n)}$ is a vector of length $n$ of 0 's except for a 1 in the $j$-th entry and

$$
P\left[J_{n+1}=j \mid \mathcal{D}^{A}(n)\right]=\frac{D_{j}^{A}(n)+\delta}{(2+\delta) n}, \quad 1 \leq j \leq n,
$$

and $L_{n+1}$ records which B.I. process in $\left\{B I_{i}\left(t-T_{i}^{A}\right): t \geq T_{i}^{A}\right\}_{1 \leq i \leq n}$ is the first to have a new birth after $T_{n}^{A}$.

When $n=1$,

$$
\widetilde{\mathcal{D}}^{A}(1)=B I_{1}(0)=2=D_{1}^{A}(1)=\mathcal{D}^{A}(1),
$$

so to prove equality in distribution for any $n$, it suffices to verify that the transition probability from $\widetilde{\mathcal{D}}(n)$ to $\widetilde{\mathcal{D}}^{A}(n+1)$ is the same as that from $\mathcal{D}^{A}(n)$ to $\mathcal{D}^{A}(n+1)$.

According to the preferential attachment setup, we have

$$
\begin{aligned}
\mathbf{P}\left(\mathcal{D}^{A}(n+1)\right. & \left.=\left(d_{1}, d_{2}, \ldots, d_{i}+1, d_{i+1}, \ldots, d_{n}, 1\right) \mid \mathcal{D}^{A}(n)=\left(d_{1}, d_{2}, \ldots, d_{n}\right)\right) \\
& =\frac{d_{i}+\delta}{(2+\delta) n}, \quad 1 \leq i \leq n .
\end{aligned}
$$


At time $T_{n}^{A}$, there are $n$ B.I. processes and each of them has a population size of $B I_{i}\left(T_{n}^{A}-T_{i}^{A}\right), 1 \leq i \leq n$. Therefore, $T_{n+1}^{A}-T_{n}^{A}$ is the minimum of $n$ independent exponential random variables, $\left\{E_{n}^{(i)}\right\}_{1 \leq i \leq n}$, with means

$$
\left(B I_{i}\left(T_{n}^{A}-T_{i}^{A}\right)+\delta\right)^{-1}, \quad 1 \leq i \leq n,
$$

which gives for any $1 \leq i \leq n$,

$$
\begin{aligned}
& \mathbf{P}\left(L_{n+1}=i \mid \widetilde{\mathcal{D}}^{A}(n)=\left(d_{1}, d_{2}, \ldots, d_{n}\right)\right) \\
& =\mathbf{P}\left(\widetilde{\mathcal{D}}^{A}(n+1)=\left(d_{1}, d_{2}, \ldots, d_{i}+1, d_{i+1}, \ldots, d_{n}, 1\right) \mid \widetilde{\mathcal{D}}^{A}(n)=\left(d_{1}, \ldots, d_{n}\right)\right) \\
& =\mathbf{P}\left(E_{n}^{(i)}<\bigwedge_{j=1, j \neq i}^{n} E_{n}^{(j)} \mid \widetilde{\mathcal{D}}^{A}(n)=\left(d_{1}, d_{2}, \ldots, d_{n}\right)\right) \\
& =\frac{B I_{i}\left(T_{n}^{A}-T_{i}^{A}\right)+\delta}{\sum_{i=1}^{n}\left(B I_{i}\left(T_{n}^{A}-T_{i}^{A}\right)+\delta\right)}=\frac{d_{i}+\delta}{(2+\delta) n} .
\end{aligned}
$$

This agrees with the transition probability in 4.5 , thus completing the proof for Model A.

For Model B, the proof follows in a similar way except that for each $n \geq 1$, $T_{n+1}^{B}-T_{n}^{B}$ is the minimum of $n+1$ independent exponential random variables with means

so that for $1 \leq i \leq n$,

$$
\left(B I_{i}\left(T_{n}^{B}-T_{i-1}^{B}\right)+\delta\right)^{-1}, \quad 1 \leq i \leq n+1,
$$

$$
\begin{aligned}
\mathbf{P}\left(\widetilde{\mathcal{D}}^{B}(n+1)\right. & \left.=\left(d_{1}, d_{2}, \ldots, d_{i}+1, d_{i+1}, \ldots, d_{n}, 1\right) \mid \widetilde{\mathcal{D}}^{B}(n)=\left(d_{1}, d_{2}, \ldots, d_{n}\right)\right) \\
& =\frac{B I_{i}\left(T_{n}^{B}-T_{i-1}^{B}\right)+\delta}{\sum_{i=1}^{n+1}\left(B I_{i}\left(T_{n}^{B}-T_{i-1}^{B}\right)+\delta\right)}=\frac{d_{i}+\delta}{(2+\delta) n+1+\delta},
\end{aligned}
$$

and

$$
\begin{aligned}
\mathbf{P}\left(\widetilde{\mathcal{D}}^{B}(n+1)\right. & \left.=\left(d_{1}, d_{2}, \ldots, d_{n}, 2\right) \mid \widetilde{\mathcal{D}}^{B}(n)=\left(d_{1}, d_{2}, \ldots, d_{n}\right)\right) \\
& =\frac{1+\delta}{(2+\delta) n+1+\delta} .
\end{aligned}
$$

Remark 3 This B.I. process construction can also be generalized for other choices of the preferential attachment functions $f$. For example, its applications to the super- and sub-linear preferential attachment models are studied in Athreya (2007).

\subsection{Asymptotic properties.}

One important reason to use the embedding technique specified in Section 4.1 is that asymptotic behavior of the degree growth in a preferential attachment model can be characterized explicitly. These asymptotic properties then help us derive weak convergence of the empirical measure, which is analogous to (1.4) in the iid case. 


\subsubsection{Branching times.}

We first consider the asymptotic behavior of the branching times $\left\{T_{n}^{A}\right\}_{n \geq 1}$ and $\left\{T_{n}^{B}\right\}_{n \geq 1}$, which are defined in Section 4.1

Proposition 4 For $\left\{T_{n}^{A}\right\}_{n \geq 1}$ and $\left\{T_{n}^{B}\right\}_{n \geq 1}$ defined in 4.3 and (4.4) respectively, we have

$$
\frac{n}{e^{(2+\delta) T_{n}^{A}}} \stackrel{\text { a.s. }}{\longrightarrow} W_{A}, \quad W_{A} \sim \operatorname{Exp}(1)
$$

and

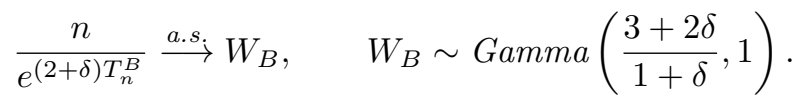

Proof Define two counting processes

$$
N_{A}(t):=\frac{1}{2} \sum_{i=1}^{\infty} B I_{i}\left(t-T_{i}^{A}\right) \mathbf{1}_{\left\{t \geq T_{i}^{A}\right\}}
$$

in Model A, and

$$
N_{B}(t):=\frac{1}{2} \sum_{i=1}^{\infty} B I_{i}\left(t-T_{i-1}^{B}\right) \mathbf{1}_{\left\{t \geq T_{i}^{B}\right\}}
$$

in Model B. In either case, we have

$$
N_{l}(t) \mathbf{1}_{\left\{t \in\left[T_{n}^{l}, T_{n+1}^{l}\right)\right\}}=n, \quad l=A, B .
$$

In other words, $\left\{T_{n}^{l}\right\}_{n \geq 1}$ are the jump times of the counting process $N_{l}(\cdot)$, for $l=A, B$, with the following structure

$$
\begin{aligned}
& \left\{T_{n+1}^{A}-T_{n}^{A}: n \geq 1\right\} \stackrel{d}{=}\left\{\frac{A_{i}}{(2+\delta) i}, i \geq 1\right\}, \\
& \left\{T_{n+1}^{B}-T_{n}^{B}: n \geq 1\right\} \stackrel{d}{=}\left\{\frac{B_{i}}{(2+\delta) i+1+\delta}, i \geq 1\right\},
\end{aligned}
$$

where $\left\{A_{i}: i \geq 1\right\}$ and $\left\{B_{i}: i \geq 1\right\}$ are iid unit exponential random variables.

From 4.8 , we see that $N_{A}(\cdot)$ is a pure birth process with $N_{A}(0)=1$ and transition rate

$$
q_{i, i+1}^{A}=(2+\delta) i, \quad i \geq 1 .
$$

Replacing $t$ with $T_{n}^{A}$ in 3.2 gives 4.6 . By $4.9, N_{B}(\cdot)$ is a B.I. process with $N_{B}(0)=1$ and transition rate $q_{i, i+1}^{B}=(2+\delta) i+1+\delta, i \geq 1$. In order to apply Theorem 1 which assumes $N_{B}(0)$, we define $N_{B}^{\prime}(t):=N_{B}(t)-1$ for all $t \geq 1$. Then $N_{B}^{\prime}$ is a B.I. process with $N_{B}^{\prime}(0)=0$ and transition rate $(2+\delta) i+3+2 \delta$, for $i \geq 0$. Therefore, 4.7) follows directly from Theorem 1 . 
4.2.2 Convergence of the measure.

Using embedding techniques, Theorem 5 gives the convergence of the empirical measure, which draws an analogy to 1.4 in the iid case.

Theorem 5 Suppose that

(1) $\left\{T_{i}^{l}: i \geq 1\right\}, l=A, B$ are distributed as in (4.8) and 4.9.

(2) $W_{l}, l=A, B$ are limit random variables as given in 4.6) and (4.7).

(3) $\left\{\sigma_{i}\right\}_{i \geq 1}$ is a sequence of independent Gamma random variables specified in (4.12 and 4.13 below.

Then in $M_{p}((0, \infty])$, we have for $\delta \geq 0$,

$$
\begin{aligned}
& \sum_{i=1}^{n} \epsilon_{D_{i}^{A}(n) / n^{1 /(2+\delta)}}(\cdot) \Rightarrow \sum_{i=1}^{\infty} \epsilon_{\sigma_{i} e^{-T_{i}^{A}} / W_{A}^{1 /(2+\delta)}}(\cdot), \\
& \sum_{i=1}^{n} \epsilon_{D_{i}^{B}(n) / n^{1 /(2+\delta)}}(\cdot) \Rightarrow \sum_{i=1}^{\infty} \epsilon_{\sigma_{i} e^{-T_{i-1}^{B} / W_{B}^{1 /(2+\delta)}}(\cdot) .}
\end{aligned}
$$

Remark 6 From 4.10a we get for any fixed $k \geq 1$, that in $\mathbb{R}_{+}^{k}$,

$$
\left(\frac{D_{(1)}^{A}(n)}{n^{1 /(2+\delta)}}, \ldots \frac{D_{(k)}^{A}(n)}{n^{1 /(2+\delta)}}\right) \Rightarrow W_{A}^{-1 /(2+\delta)}\left(\left(\sigma \cdot e^{-T_{\cdot}^{A}}\right)_{(1)}, \ldots,\left(\sigma \cdot e^{-T^{A}}\right)_{(k)}\right)
$$

where a subscript inside parentheses indicates ordering so that $D_{(1)}^{A}(n) \geq \cdots \geq$ $D_{(k)}^{A}$ and the limit on the right side of 4.11 represents the ordered $k$ largest points from the right side of 4.10a). A similar result for Model B follows from $4.10 \mathrm{~b}$.

To prove Theorem 5, we first need to show the following lemma, which gives the asymptotic limit of the degree sequence under the B.I. process framework.

Lemma 7 Suppose that

(1) $\left\{T_{i}^{l}: i \geq 1\right\}, l=A, B$ are distributed as in 4.8 and 4.9.

(2) $W_{l}, l=A, B$ are limit random variables as given in 4.6) and (4.7).

Then we have the following convergence results pertinent to the degree sequence $\left\{D_{i}^{l}(n): 1 \leq i \leq n\right\}$, for $l=A, B$ :

(i) For each $i \geq 1$,

$$
\begin{gathered}
\frac{B I_{i}\left(T_{n}^{A}-T_{i}^{A}\right)}{e^{T_{n}^{A}}} \stackrel{a . s}{\longrightarrow} \sigma_{i} e^{-T_{i}^{A}}, \\
\frac{B I_{i}\left(T_{n}^{B}-T_{i-1}^{B}\right)}{e^{T_{n}^{B}}} \stackrel{a . s .}{\longrightarrow} \sigma_{i} e^{-T_{i-1}^{B}},
\end{gathered}
$$

where $\left\{\sigma_{i}\right\}_{i \geq 1}$ are a sequence of independent Gamma random variables with

$$
\sigma_{1} \sim \operatorname{Gamma}(2+\delta, 1), \quad \text { and } \sigma_{i} \sim \operatorname{Gamma}(1+\delta, 1), \quad i \geq 2 .
$$

Furthermore, for $i \geq 1, \sigma_{i} \Perp e^{-T_{i}^{A}}$ in Model $A$ and $\sigma_{i} \Perp e^{-T_{i-1}^{B}}$ in Model $B$. 
(ii) For $\delta>-1$,

$$
\begin{aligned}
& \max _{i \geq 1} \frac{D_{i}^{A}(n)}{n^{1 /(2+\delta)}} \stackrel{P}{\rightarrow} W_{A}^{-1 /(2+\delta)} \max _{i \geq 1} \sigma_{i} e^{-T_{i}^{A}}, \\
& \max _{i \geq 1} \frac{D_{i}^{B}(n)}{n^{1 /(2+\delta)}} \stackrel{P}{\rightarrow} W_{B}^{-1 /(2+\delta)} \max _{i \geq 1} \sigma_{i} e^{-T_{i-1}^{B}},
\end{aligned}
$$

where we set $T_{0}^{B}:=0$ and $D_{i}^{l}(n):=0$ for all $i \geq n+1, l=A, B$.

Proof (i) For the B.I. processes $\left\{B I_{i}(\cdot)\right\}_{i \geq 1}$ defined here, all of them have initial values greater than 0 . Hence, in order to apply the asymptotic results in Tavaré (1987), we need to modify them such that they all start with 0 . To do this, set for all $t \geq 0$,

$$
B I_{1}^{\prime}(t):=B I_{1}(t)-2, \quad B I_{i}^{\prime}(t):=B I_{i}(t)-1, \quad i \geq 2,
$$

and we have $B I_{i}^{\prime}(0)=0$ for all $i \geq 0$. The transition rate needs to be changed accordingly, i.e. the process $B I_{1}^{\prime}(\cdot)$ has transition rate $q_{j, j+1}^{\prime}=j+2+\delta$ and that for $B I_{i}^{\prime}(\cdot), i \geq 2$, becomes $j+1+\delta, j \geq 0$.

Throughout the rest of the proof of Lemma 7, we only show the case for Model A and the result for Model B follows from the same argument. Now applying Theorem 1 gives that as $t \rightarrow \infty$,

$$
\frac{B I_{i}\left(t-T_{i}^{A}\right)}{e^{t-T_{i}^{A}}} \stackrel{\text { a.s. }}{\longrightarrow} \sigma_{i}, \quad i \geq 1,
$$

where $\left\{\sigma_{i}\right\}_{i \geq 1}$ are independent Gamma random variables with

$$
\sigma_{1} \sim \operatorname{Gamma}(2+\delta, 1) \quad \text { and } \quad \sigma_{i} \sim \operatorname{Gamma}(1+\delta, 1), \quad i \geq 2 .
$$

Thus as $n \rightarrow \infty$,

$$
\frac{B I_{i}\left(T_{n}^{l}-T_{i}^{A}\right)}{e^{T_{n}^{l}-T_{i}^{A}}} \stackrel{\text { a.s. }}{\longrightarrow} \sigma_{i}, \quad i \geq 1
$$

which gives 4.12a.

For $i \geq 2$, the independence of $\sigma_{i}$ and $T_{i}^{A}$ follows from the construction and this completes the proof of (i).

(ii) Combining (4.12a) with 4.6), we have for fixed $1 \leq i \leq n$,

$$
\frac{B I_{i}\left(T_{n}^{A}-T_{i}^{A}\right)}{n^{1 /(2+\delta)}} \stackrel{\text { a.s. }}{\longrightarrow} \frac{\sigma_{i} e^{-T_{i}^{A}}}{W_{A}^{1 /(2+\delta)}},
$$

and $B I_{i}\left(T_{n}^{A}-T_{i}^{A}\right)=0$ for $i \geq n+1$. By Theorem 2 it suffices to show

$$
\max _{i \geq 1} \frac{B I_{i}\left(T_{n}^{A}-T_{i}^{A}\right)}{n^{1 /(2+\delta)}} \stackrel{\text { a.s. }}{\longrightarrow} \max _{i \geq 1} \frac{\sigma_{i} e^{-T_{i}^{A}}}{W_{A}^{1 /(2+\delta)}},
$$

which is proved in Athreya et al. (2008, Theorem 1.1(iii)). 
With the preparation in Lemma 7 , we are ready to prove the convergence result in Theorem 5 .

Proof of Theorem 5. Note that the limit random variables

$$
\sigma_{i} e^{-T_{i}^{A}} W_{A}^{-1 /(2+\delta)}, \quad i \geq 1,
$$

have continuous distributions, so for any $y>0$,

$$
\mathbf{P}\left(\sum_{i=1}^{\infty} \epsilon_{\sigma_{i} e^{-T_{i}^{A}} / W_{A}^{1 /(2+\delta)}}(\{y\})=0\right)=1 .
$$

Hence, by Kallenberg's theorem for weak convergence to a point process on an interval (see Kallenberg (2017, Theorem 4.18) and Resnick (1987, Proposition 3.22)), proving (4.10a) requires checking

(a) For $y>0$, as $n \rightarrow \infty$,

$$
\mathbf{E}\left(\sum_{i=1}^{n} \epsilon_{D_{i}^{A}(n) / n^{1 /(2+\delta)}}(y, \infty]\right) \rightarrow \mathbf{E}\left(\sum_{i=1}^{\infty} \epsilon_{\sigma_{i} e^{-T_{i}^{A}} / W_{A}^{1 /(2+\delta)}}(y, \infty]\right) .
$$

(b) For $y>0$, as $n \rightarrow \infty$,

$$
\begin{aligned}
& \mathbf{P}\left(\sum_{i=1}^{n} \epsilon_{D_{i}^{A}(n) / n^{1 /(2+\delta)}}(y, \infty]=0\right) \\
& \longrightarrow \mathbf{P}\left(\sum_{i=1}^{\infty} \epsilon_{\sigma_{i} e^{-T_{i}^{A}} / W_{A}^{1 /(2+\delta)}}(y, \infty]=0\right) .
\end{aligned}
$$

To show 4.15, first note that for any $M>0$,

$$
\begin{aligned}
\mathbf{E}\left(\sum_{i=1}^{M} \epsilon_{D_{i}^{A}(n) / n^{1 /(2+\delta)}}(y, \infty]\right) & =\sum_{i=1}^{M} \mathbf{P}\left(\frac{D_{i}^{A}(n)}{n^{1 /(2+\delta)}}>y\right) \\
& \longrightarrow \sum_{i=1}^{M} \mathbf{P}\left(\sigma_{i} e^{-T_{i}^{A}} W_{A}^{-1 /(2+\delta)}>y\right) \\
& =\mathbf{E}\left(\sum_{i=1}^{M} \epsilon_{\sigma_{i} e^{-T_{i}^{A} / W_{A}^{1 /(2+\delta)}}}(y, \infty]\right),
\end{aligned}
$$

as $n \rightarrow \infty$. By Chebyshev's inequality we have for any $k>2+\delta$,

$$
\begin{aligned}
\mathbf{E}\left(\sum_{i=M+1}^{n} \epsilon_{D_{i}^{A}(n) / n^{1 /(2+\delta)}}(y, \infty]\right) & =\sum_{i=M+1}^{n} \mathbf{P}\left(\frac{D_{i}^{A}(n)}{n^{1 /(2+\delta)}}>y\right) \\
& \leq y^{-k} \sum_{i=M+1}^{n} \mathbf{E}\left[\left(\frac{D_{i}^{A}(n)}{n^{1 /(2+\delta)}}\right)^{k}\right] .
\end{aligned}
$$


Also, we have for $\delta \geq 0$,

$$
\mathbf{E}\left[\left(\frac{D_{i}^{A}(n)}{n^{1 /(2+\delta)}}\right)^{k}\right] \leq \mathbf{E}\left[\left(\frac{D_{i}^{A}(n)+\delta}{n^{1 /(2+\delta)}}\right)^{k}\right] \leq \mathbf{E}\left[\left(\frac{\sigma_{i} e^{-T_{i}^{A}}}{W_{A}^{1 /(2+\delta)}}\right)^{k}\right],
$$

where the last inequality follows from the result in van der Hofstad (2017. Equation (8.7.26)). From van der Hofstad (2017, Equation (8.7.22)), we have

$$
\mathbf{E}\left[\left(\frac{\sigma_{i} e^{-T_{i}^{A}}}{W_{A}^{1 /(2+\delta)}}\right)^{k}\right]=\frac{\Gamma\left(i-\frac{1}{2+\delta}\right)}{\Gamma\left(i+\frac{k-1}{2+\delta}\right)} \frac{\Gamma(k+1+\delta)}{\Gamma(1+\delta)} \sim C_{k, \delta} i^{-\frac{k}{2+\delta}},
$$

for $i$ large and $C_{k, \delta}>0$. Hence, continuing from (4.17), we have

$$
\begin{aligned}
\mathbf{E}\left(\sum_{i=M+1}^{n} \epsilon_{D_{i}^{A}(n) / n^{1 /(2+\delta)}}(y, \infty]\right) & \leq y^{-k} \sum_{i=M+1}^{n} \mathbf{E}\left[\left(\frac{D_{i}^{A}(n)}{n^{1 /(2+\delta)}}\right)^{k}\right] \\
& \leq y^{-k} \sum_{i=M+1}^{\infty} \mathbf{E}\left[\left(\frac{\sigma_{i} e^{-T_{i}^{A}}}{W_{A}^{1 /(2+\delta)}}\right)^{k}\right] \\
& =y^{-k} \sum_{i=M+1}^{\infty} \frac{\Gamma\left(i-\frac{1}{2+\delta}\right)}{\Gamma\left(i+\frac{k-1}{2+\delta}\right)} \frac{\Gamma(k+1+\delta)}{\Gamma(1+\delta)} \\
& \stackrel{M \rightarrow \infty}{\longrightarrow} 0,
\end{aligned}
$$

since $k /(2+\delta)>1$. This verifies Condition (a).

To see (4.16), we have

$$
\begin{aligned}
\left\{\sum_{i=1}^{n} \epsilon_{D_{i}^{A}(n) / n^{1 /(2+\delta)}}(y, \infty]=0\right\} & =\left\{\frac{D_{i}^{A}(n)}{n^{1 /(2+\delta)}} \leq y, 1 \leq i \leq n\right\} \\
& =\left\{\max _{1 \leq i \leq n} \frac{D_{i}^{A}(n)}{n^{1 /(2+\delta)}} \leq y\right\} .
\end{aligned}
$$

Since we set $D_{i}^{A}(n)=0$ for all $i \geq n+1$, then

$$
\left\{\max _{1 \leq i \leq n} \frac{D_{i}^{A}(n)}{n^{1 /(2+\delta)}} \leq y\right\}=\left\{\max _{i \geq 1} \frac{D_{i}^{A}(n)}{n^{1 /(2+\delta)}} \leq y\right\} .
$$

Similarly,

$$
\left\{\sum_{i=1}^{\infty} \epsilon_{\sigma_{i} e^{-T_{i}^{A}} / W_{A}^{1 /(2+\delta)}}(y, \infty]=0\right\}=\left\{\max _{i \geq 1} \frac{\sigma_{i} e^{-T_{i}^{A}}}{W_{A}^{1 /(2+\delta)}} \leq y\right\} .
$$

By 4.14a, we have for $y>0$,

$$
\mathbf{P}\left(\max _{i \geq 1} \frac{D_{i}^{A}(n)}{n^{1 /(2+\delta)}} \leq y\right) \rightarrow \mathbf{P}\left(\max _{i \geq 1} \frac{\sigma_{i} e^{-T_{i}^{A}}}{W_{A}^{1 /(2+\delta)}} \leq y\right), \quad \text { as } n \rightarrow \infty,
$$

which gives 4.16 and completes the proof of (iv). 


\section{Consistency of Hill Estimator.}

We now turn to (1.8) as preparation for considering consistency of the Hill estimator. We first give a plausibility argument based on the form of the limit point measure in (4.10a) or 4.10b). However, proving (1.8) requires showing $N_{>k}(n) / n$ concentrates on $p_{>k}$, for all $k \geq 1$, which in other words means controlling the bias for $N_{>k}(n) / n$ and the discrepancy between $E\left(N_{>k}(n) / n\right)$ and $p_{>k}$. Later we will show this is true for our Model A but we were not successful for Model B. See Remark 9

\subsection{Heuristics.}

Before starting formalities, here is a heuristic explanation for the consistency of the Hill estimator when applied to preferential attachment data from Model A. The heuristic is the same for both Model A and B so for simplicity, we focus on Model A and apply the Hill estimator to the limit points in 4.10a). Since the Gamma random variables $\sigma_{i}$ have light tailed distributions, one may expect that $\left\{\sigma_{i}: i \geq 1\right\}$ will not distort the consistency result and so we pretend the $\sigma_{i}$ 's are absent; then what remains in the limit points is monotone in $i$. Set $Y_{i}:=e^{-T_{i}^{A}} / W_{A}^{1 /(2+\delta)}$ and apply the Hill estimator to the $Y^{\prime} s$ to get

$H_{k, n}=\frac{1}{k} \sum_{i=1}^{k} \log \left(\frac{Y_{i}}{Y_{k+1}}\right)=\frac{1}{k} \sum_{i=1}^{k}\left(T_{k+1}^{A}-T_{i}^{A}\right)$.

Recall from just after 4.5 that

$$
T_{n+1}^{A}-T_{n}^{A} \stackrel{d}{=} E_{n} /(n(2+\delta)),
$$

where $E_{n}, n \geq 1$ are iid unit exponential random variables. Then

$$
H_{k, n}=\frac{1}{k} \sum_{i=1}^{k} \sum_{l=i}^{k}\left(T_{l+1}^{A}-T_{l}^{A}\right)=\frac{1}{k} \sum_{l=1}^{k} l\left(T_{l+1}^{A}-T_{l}^{A}\right)=\frac{1}{k} \sum_{l=1}^{k} \frac{E_{l}}{2+\delta} \stackrel{\text { a.s. }}{\longrightarrow} \frac{1}{2+\delta},
$$

by strong law of large numbers, provided that $k \rightarrow \infty$.

There are clear shortcomings to this approach, the most obvious being that we only dealt with the points at asymptopia rather than $\left\{D_{i}(n), 1 \leq i \leq n\right\}$. Furthermore we simplified the limit points by neglecting the $\sigma_{i}$ 's. We have not found an effective way to analyze order statistics of $\left\{\sigma_{i} e^{-T_{i}^{A}} / W_{A}^{1 /(2+\delta)}: i \geq 1\right\}$.

Concentration results for degree counts provide a traditional tool to prove (1.8) and we pursue this for Model A in the next subsection.

\subsection{Concentration of the degree sequence in Model A}

We begin with considering the sequence of degree counts $\left\{N_{>k}(n)\right\}_{k \geq 1}$. Theorem 8 shows that $N_{>k}(n) / n$ concentrates on $p_{>k}$, for all $k \geq 1$. This concentration is what is needed for the consistency of the Hill estimator for network 
data. Note that for the linear preferential attachment model, the concentration result for $N_{k}(n)$ is known from van der Hofstad (2017, Theorem 8.3).

Theorem 8 For $\delta>-1$ there exists a constant $C>0$, such that as $n \rightarrow \infty$,

$$
\boldsymbol{P}\left(\max _{k}\left|N_{>k}(n)-n p_{>k}\right| \geq C(1+\sqrt{n \log n})\right)=o(1) .
$$

Proof Let $\mu_{>k}(n):=\mathbf{E}\left(N_{>k}(n)\right)$. Following the proof in van der Hofstad $(2017$ Proposition 8.4), we have for any $C_{\mu}>2 \sqrt{2}$,

$$
\mathbf{P}\left(\left|N_{>k}(n)-\mu_{>k}(n)\right| \geq C_{\mu} \sqrt{n \log n}\right)=o(1 / n) .
$$

Since $N_{>k}(n)=0$ a.s. for all $k>n$, then

$$
\begin{aligned}
& \mathbf{P}\left(\max _{k}\left|N_{>k}(n)-\mu_{>k}(n)\right| \geq C_{\mu} \sqrt{n \log n}\right) \\
& =\mathbf{P}\left(\max _{0 \leq k \leq n}\left|N_{>k}(n)-\mu_{>k}(n)\right| \geq C_{\mu} \sqrt{n \log n}\right) \\
& \leq \sum_{k=1}^{n} \mathbf{P}\left(\left|N_{>k}(n)-\mu_{>k}(n)\right| \geq C_{\mu} \sqrt{n \log n}\right)=o(1) .
\end{aligned}
$$

Note that (5.2) also holds for Model B, but we do not succeed in proving the concentration result later in 5.3 for Model B; see Remark 9 for details. We are now left to show the concentration of $\mu_{>k}(n)$ on $n p_{>k}$ in the setup of Model A. We claim that

$$
\left|\mu_{>k}(n)-n p_{>k}\right| \leq C^{\prime}, \quad \forall n \geq 1, \quad \forall k \geq 1 .
$$

for some constant $C^{\prime}>0$ specified later. We prove 5.3 by induction. First, by model construction, $N_{>k}(n)$ satisfies

$$
\begin{aligned}
\mathbf{E}\left(N_{>k}(n+1) \mid G(n)\right) & =N_{>k}(n)+\frac{k+\delta}{(2+\delta) n} N_{k}(n) \\
& =N_{>k}(n)+\frac{k+\delta}{(2+\delta) n}\left(N_{>k-1}(n)-N_{>k}(n)\right), \quad k \geq 1 .
\end{aligned}
$$

Therefore,

$$
\mu_{>k}(n+1)=\mu_{>k}(n)+\frac{k+\delta}{(2+\delta) n}\left(\mu_{>k-1}(n)-\mu_{>k}(n)\right), \quad k \geq 1 .
$$

Moreover, it follows from 2.5 and 2.6 that

$$
p_{>k}=\frac{k+\delta}{2+\delta} p_{k}, \quad k \geq 1
$$

Thus $p_{>k}$ satisfies the recursion

$$
p_{>k}=\frac{k+\delta}{2+\delta}\left(p_{>k-1}-p_{>k}\right), \quad k \geq 1,
$$


since $p_{k}=p_{>k-1}-p_{>k}$. Let $\varepsilon_{>k}(n):=\mu_{>k}(n)-n p_{>k}$, then 5.4 and 5.5 give that for $k \geq 1$,

$$
\varepsilon_{>k}(n+1)=\left(1-\frac{k+\delta}{(2+\delta) n}\right) \varepsilon_{>k}(n)+\frac{k+\delta}{(2+\delta) n} \varepsilon_{>k-1}(n) .
$$

In order to prove 5.3 , we initiate the induction procedure by first inducting on $n$ to prove

$$
\left|\varepsilon_{>1}(n)\right| \leq 1, \quad \forall n \geq 1 .
$$

When $n=1$, the graph $G(1)$ consists of one node and $D_{1}(1)=2$. Since $p_{>k} \leq 1$, we have

$$
\left|\varepsilon_{>k}(1)\right|=\left|\mu_{>k}(1)-p_{>k}\right| \leq 1, \quad \forall k \geq 1,
$$

which also implies $\left|\varepsilon_{>1}(1)\right| \leq 1$. Assume $\left|\varepsilon_{>1}(n)\right| \leq 1$ and we want to show $\left|\varepsilon_{>1}(n+1)\right| \leq 1$. Note that $\varepsilon_{>0}(n)=\mu_{>0}(n)-n p_{>0}=n-n \cdot 1=0$, then when $k=1$, (5.6) becomes

$$
\varepsilon_{>1}(n+1)=\left(1-\frac{1+\delta}{(2+\delta) n}\right) \varepsilon_{>1}(n),
$$

and $1-\frac{1+\delta}{(2+\delta) n} \geq 0$ for $n \geq 1$. This gives

$$
\left|\varepsilon_{>1}(n+1)\right| \leq\left(1-\frac{1+\delta}{(2+\delta) n}\right)\left|\varepsilon_{>1}(n)\right| \leq 1 .
$$

Hence, (5.7) is verified, which gives the initialization step of the induction.

Since proving (5.3) requires showing

$$
\sup _{n \geq 1}\left|\varepsilon_{>k}(n)\right| \leq C_{p}, \quad \forall k \geq 1,
$$

for some constant $C_{p}$ which will be defined later, we verify 5.9 by inducting on $k$. What is proved in (5.7) gives the initialization of the induction $(k=1)$ and we want to verify

$$
\left|\varepsilon_{>k}(n)\right| \leq C_{p}, \quad \forall n \geq 1
$$

assuming

$$
\left|\varepsilon_{>k-1}(n)\right| \leq C_{p}, \quad \forall n \geq 1,
$$

for some $k \geq 2$. To do this, we again use induction on $n$, with the result for the base case $n=1$ being verified in $(5.8)$. We now need to show $\left|\varepsilon_{>k}(n+1)\right| \leq C_{p}$, given both $\left|\varepsilon_{>k}(n)\right| \leq C_{p}$ and $(5.10)$.

The recursion in $(5.6)$ gives that for $2 \leq k \leq(2+\delta) n-\delta$,

$$
\left|\varepsilon_{>k}(n+1)\right| \leq\left(1-\frac{k+\delta}{(2+\delta) n}\right)\left|\varepsilon_{>k}(n)\right|+\frac{k+\delta}{(2+\delta) n}\left|\varepsilon_{>k-1}(n)\right| \leq 1 .
$$

For $k>(2+\delta) n-\delta$,

$$
\left|\varepsilon_{>k}(n+1)\right|=(n+1) p_{>k} .
$$


Since $(2+\delta) n-\delta \geq n+1$ for $\delta>-1, n \geq 1$, we apply 2.6 and there exists a $C_{p}=C_{p}(\delta)$ such that

$$
p_{>k} \leq C_{p}(n+1)^{-(2+\delta)}
$$

which gives

$$
\left|\varepsilon_{>k}(n+1)\right|=(n+1) p_{>k} \leq C_{p}(n+1)^{-(1+\delta)} \leq C_{p} .
$$

Thus, the claim in 5.3 is verified with

$$
C^{\prime}:=\max \left\{1, C_{p}\right\}
$$

With the result in 5.2 , the proof of the theorem is complete by choosing $C=\max \left\{C_{\mu}, C^{\prime}\right\}$.

Remark 9 The induction argument does not suffice to prove (5.3) for Model B. To see this, we re-compute the recursion on the difference term $\varepsilon_{>k}(n)$ for Model B and (5.6) then becomes

$$
\begin{aligned}
\varepsilon_{>k}(n+1)= & \left(1-\frac{k+\delta}{(2+\delta) n+1+\delta}\right) \varepsilon_{>k}(n)+\frac{k+\delta}{(2+\delta) n+1+\delta} \varepsilon_{>k-1}(n) \\
& +\left(\frac{1}{2+\delta}-\frac{n}{(2+\delta) n+1+\delta}\right)(k+\delta)\left(p_{>k-1}-p_{>k}\right) \\
= & \left(1-\frac{k+\delta}{(2+\delta) n+1+\delta}\right) \varepsilon_{>k}(n)+\frac{k+\delta}{(2+\delta) n+1+\delta} \varepsilon_{>k-1}(n) \\
& +\frac{1+\delta}{2+\delta} \frac{(k+\delta) p_{k}}{(2+\delta) n+1+\delta} .
\end{aligned}
$$

By van der Hofstad (2017, Exercise 8.19), $(k+\delta) p_{k} \leq 2+\delta$. Therefore, if $\left|\varepsilon_{>k}(n)\right| \leq 1$, then

$$
\left|\varepsilon_{>k}(n+1)\right| \leq 1+\frac{1}{n+1},
$$

which contradicts the induction hypothesis.

Since the concentration inequality proved in Theorem 8 cannot be validated for Model B by induction, we are also not able to verify the consistency of the Hill estimator in Model B, using the proof steps proposed here. This will be deferred as future research.

\subsection{Convergence of the tail empirical measure for Model A}

We then use the concentration result in (5.1) to analyze the convergence of the tail empirical measure. First consider the degree of each node in $G(n)$,

$$
\left(D_{1}(n), D_{2}(n), \ldots, D_{n}(n)\right)
$$


and let

$$
D_{(1)}(n) \geq D_{(2)}(n) \geq \cdots \geq D_{(n)}(n)
$$

be the corresponding order statistics. Then the tail empirical measure becomes

$$
\hat{\nu}_{n}(\cdot):=\frac{1}{k_{n}} \sum_{i=1}^{n} \epsilon_{D_{i}(n) / D_{\left(k_{n}\right)}(n)}(\cdot),
$$

for some intermediate sequence $\left\{k_{n}\right\}$, i.e. $k_{n} \rightarrow \infty$ and $k_{n} / n \rightarrow 0$ as $n \rightarrow \infty$.

Theorem 10 Suppose that $\left\{k_{n}\right\}$ is some intermediate sequence satisfying

$$
\liminf _{n \rightarrow \infty} k_{n} /(n \log n)^{1 / 2}>0 \quad \text { and } \quad k_{n} / n \rightarrow 0 \quad \text { as } n \rightarrow \infty,
$$

then

$$
\hat{\nu}_{n} \Rightarrow \nu_{2+\delta},
$$

in $M_{+}((0, \infty])$, where $\nu_{2+\delta}(x, \infty]=x^{-(2+\delta)}, x>0$.

Proof Step 1. We first show that for fixed $t>0$,

$$
\frac{D_{\left(\left[k_{n} t\right]\right)}(n)}{b\left(n / k_{n}\right)} \stackrel{P}{\rightarrow} t^{-\frac{1}{2+\delta}}
$$

where

$$
b\left(n / k_{n}\right)=\left(\frac{\Gamma(3+2 \delta)}{\Gamma(1+\delta)}\right)^{\frac{1}{2+\delta}}\left(n / k_{n}\right)^{\frac{1}{2+\delta}}
$$

Since

$$
\begin{aligned}
& \mathbf{P}\left(\left|\frac{D_{\left(\left[k_{n} t\right]\right)}(n)}{b\left(n / k_{n}\right)}-t^{-\frac{1}{2+\delta}}\right|>\epsilon\right) \\
& \leq \mathbf{P}\left(D_{\left(\left[k_{n} t\right]\right)}(n)>b\left(n / k_{n}\right)\left(t^{-\frac{1}{2+\delta}}+\epsilon\right)\right)+\mathbf{P}\left(D_{\left(\left[k_{n} t\right]\right)}(n)<b\left(n / k_{n}\right)\left(t^{-\frac{1}{2+\delta}}-\epsilon\right)\right) \\
& =: I+I I,
\end{aligned}
$$

it suffices to show $I, I I \rightarrow 0$ as $n \rightarrow \infty$. For the first term, we have, with $u_{t}:=t^{-\frac{1}{2+\delta}}+\epsilon$,

$$
\begin{aligned}
& I \leq \mathbf{P}\left(N_{>\left[b\left(n / k_{n}\right) u_{t}\right]}(n) \geq\left[k_{n} t\right]\right) \\
& =\mathbf{P}\left(N_{>\left[b\left(n / k_{n}\right) u_{t}\right]}(n)-n p_{>\left[b\left(n / k_{n}\right) u_{t}\right]} \geq\left[k_{n} t\right]-n p_{>\left[b\left(n / k_{n}\right) u_{t}\right]}\right) .
\end{aligned}
$$

Using Stirling's formula, van der Hofstad (2017, Equation 8.3.9) gives

$$
\frac{\Gamma(t+a)}{\Gamma(t)}=t^{a}(1+O(1 / t))
$$

Recall the definition of $p_{>k}$ in $(2.6)$ for fixed $k$, then we have

$$
\frac{n}{k_{n}} p_{>\left[b\left(n / k_{n}\right) y\right]}=\frac{n}{k_{n}} \frac{\Gamma(3+2 \delta)}{\Gamma(1+\delta)} \frac{\Gamma\left(\left[b\left(n / k_{n}\right) y\right]+1+\delta\right)}{\Gamma\left(\left[b\left(n / k_{n}\right) y\right]+3+2 \delta\right)}
$$




$$
\begin{aligned}
& =\frac{\Gamma(3+2 \delta)}{\Gamma(1+\delta)} \frac{n}{k_{n}}\left(b\left(n / k_{n}\right) y\right)^{-(2+\delta)}\left(1+O\left(\frac{1}{b\left(n / k_{n}\right)}\right)\right) \\
& =y^{-(2+\delta)}\left(1+O\left(\frac{1}{b\left(n / k_{n}\right)}\right)\right) .
\end{aligned}
$$

Continuing from (5.14) then gives

$$
\begin{aligned}
& I \leq \mathbf{P}\left(N_{>\left[b\left(n / k_{n}\right) u_{t}\right]}(n)-n p_{>\left[b\left(n / k_{n}\right) u_{t}\right]} \geq\left[k_{n} t\right]-n p_{>\left[b\left(n / k_{n}\right) u_{t}\right]}\right) \\
& \leq \mathbf{P}\left(\left|N_{>\left[b\left(n / k_{n}\right) u_{t}\right]}(n)-n p_{>\left[b\left(n / k_{n}\right) u_{t}\right]}\right| \geq k_{n}\left(t-u_{t}^{-(2+\delta)}+O\left(1 / b\left(n / k_{n}\right)\right)\right)\right) \\
& \leq \mathbf{P}\left(\max _{j}\left|N_{>j}(n)-n p_{>j}\right| \geq k_{n}\left(t-u_{t}^{-(2+\delta)}+O\left(1 / b\left(n / k_{n}\right)\right)\right)\right) .
\end{aligned}
$$

By Theorem 8 , the right hand side goes to 0 as $n \rightarrow \infty$, provided that $k_{n}$ satisfies (5.11). Similarly, we can also show $I I \rightarrow 0$ as $n \rightarrow \infty$ for $k_{n}$ satisfying (5.11), thus proving (5.13).

Step 2. Note that $D_{\left(\left[k_{n} t\right]\right)}(n)$ is decreasing in $t$ and the limit in 5.13$)$ is continuous on $(0, \infty]$, which implies

$$
\frac{D_{\left(\left[k_{n} t\right]\right)}(n)}{b\left(n / k_{n}\right)} \stackrel{P}{\rightarrow} t^{-\frac{1}{2+\delta}}, \quad \text { in } D(0, \infty] .
$$

This gives, by inversion and Resnick 2007, Proposition 3.2),

$$
\frac{1}{k_{n}} \sum_{i=1}^{n} \epsilon_{D_{i}(n) / b\left(n / k_{n}\right)}(t, \infty] \stackrel{P}{\rightarrow} t^{-(2+\delta)}, \quad t \in(0, \infty],
$$

in $D(0, \infty]$. Moreover,

$$
\left(\frac{1}{k_{n}} \sum_{i=1}^{n} \epsilon_{D_{i}(n) / b\left(n / k_{n}\right)}, \frac{D_{\left(\left[k_{n}\right]\right)}(n)}{b\left(n / k_{n}\right)}\right) \Rightarrow\left(\nu_{2+\delta}, 1\right)
$$

in $M_{+}((0, \infty]) \times(0, \infty)$.

Step 3. With 5.16, we use a scaling argument to prove 5.12). Define the operator

$$
S: M_{+}((0, \infty]) \times(0, \infty) \mapsto M_{+}((0, \infty])
$$

by

$$
S(\nu, c)(A)=\nu(c A) .
$$

By the proof in Resnick (2007, Theorem 4.2), the mapping $S$ is continuous at $\left(\nu_{2+\delta}, 1\right)$. Therefore, applying the continuous mapping $S$ to the joint weak convergence in 5.17) gives 5.12. 
5.4 Consistency of the Hill estimator for Model A

We are now able to prove the consistency of the Hill estimator applied to $\left\{D_{i}(n): 1 \leq i \leq n\right\}$, i.e.

$$
H_{k_{n}, n}=\frac{1}{k_{n}} \sum_{i=1}^{k_{n}} \log \frac{D_{(i)}(n)}{D_{\left(k_{n}+1\right)}(n)} .
$$

Theorem 11 Let $\left\{k_{n}\right\}$ be an intermediate sequence satisfying (5.11), then

$$
H_{k_{n}, n} \stackrel{P}{\rightarrow} \frac{1}{2+\delta}
$$

Proof First define a mapping $T: D(0, \infty] \mapsto \mathbb{R}_{+}$by

$$
T(f)=\int_{1}^{\infty} f(y) \frac{\mathrm{d} y}{y},
$$

and note that

$$
H_{k_{n}, n}=\int_{1}^{\infty} \hat{\nu}_{n}(y, \infty] \frac{\mathrm{d} y}{y} .
$$

Therefore, proving the consistency of $H_{k_{n}, n}$ requires justifying the continuity of the mapping $T$ at $\nu_{2+\delta}$, so that

$$
H_{k_{n}, n}=\int_{1}^{\infty} \hat{\nu}_{n}(y, \infty] \frac{\mathrm{d} y}{y} \stackrel{P}{\rightarrow} \int_{1}^{\infty} \nu_{2+\delta}(y, \infty] \frac{\mathrm{d} y}{y}=\frac{1}{2+\delta} .
$$

Note that for any $M$ we have

$$
\int_{1}^{M} \hat{\nu}_{n}(y, \infty] \frac{\mathrm{d} y}{y} \stackrel{P}{\rightarrow} \int_{1}^{M} \nu_{2+\delta}(y, \infty] \frac{\mathrm{d} y}{y},
$$

so we only need to show

$$
\int_{M}^{\infty} \hat{\nu}_{n}(y, \infty] \frac{\mathrm{d} y}{y} \stackrel{P}{\rightarrow} \int_{M}^{\infty} \nu_{2+\delta}(y, \infty] \frac{\mathrm{d} y}{y} .
$$

By the second converging together theorem (see Resnick (2007, Theorem 3.5)), it suffices to show

$$
\lim _{M \rightarrow \infty} \limsup _{n \rightarrow \infty} \mathbf{P}\left(\int_{M}^{\infty} \hat{\nu}_{n}(y, \infty] \frac{\mathrm{d} y}{y}>\varepsilon\right)=0 .
$$

Consider the probability in 5.18 and we have

$$
\begin{aligned}
& \mathbf{P}\left(\int_{M}^{\infty} \hat{\nu}_{n}(y, \infty] \frac{\mathrm{d} y}{y}>\varepsilon\right) \\
& \leq \mathbf{P}\left(\int_{M}^{\infty} \hat{\nu}_{n}(y, \infty] \frac{\mathrm{d} y}{y}>\varepsilon,\left|\frac{D_{\left(k_{n}\right)}(n)}{b\left(n / k_{n}\right)}-1\right|<\eta\right)
\end{aligned}
$$




$$
\begin{aligned}
& +\mathbf{P}\left(\int_{M}^{\infty} \hat{\nu}_{n}(y, \infty] \frac{\mathrm{d} y}{y}>\varepsilon,\left|\frac{D_{\left(k_{n}\right)}(n)}{b\left(n / k_{n}\right)}-1\right| \geq \eta\right) \\
\leq & \mathbf{P}\left(\int_{M}^{\infty} \frac{1}{k_{n}} \sum_{i=1}^{n} \epsilon_{D_{i}(n) / b\left(n / k_{n}\right)}((1-\eta) y, \infty] \frac{\mathrm{d} y}{y}>\varepsilon\right) \\
+ & \mathbf{P}\left(\left|\frac{D_{\left(k_{n}\right)}(n)}{b\left(n / k_{n}\right)}-1\right| \geq \eta\right)=: A+B .
\end{aligned}
$$

By (5.13), $B \rightarrow 0$ as $n \rightarrow \infty$, and using the Markov inequality, $A$ is bounded by

$$
\begin{aligned}
& \frac{1}{\varepsilon} \mathbf{E}\left(\int_{M}^{\infty} \frac{1}{k_{n}} \sum_{i=1}^{n} \epsilon_{D_{i}(n) / b\left(n / k_{n}\right)}((1-\eta) y, \infty] \frac{\mathrm{d} y}{y}\right) \\
& =\frac{1}{\varepsilon} \mathbf{E}\left(\int_{M(1-\eta)}^{\infty} \frac{1}{k_{n}} \sum_{i=1}^{n} \epsilon_{D_{i}(n) / b\left(n / k_{n}\right)}(y, \infty] \frac{\mathrm{d} y}{y}\right) \\
& \leq \frac{1}{\varepsilon} \int_{M(1-\eta)}^{\infty} \frac{1}{k_{n}} \mathbf{E}\left(N_{>\left[b\left(n / k_{n}\right) y\right]}(n)\right) \frac{\mathrm{d} y}{y} .
\end{aligned}
$$

Furthermore, we also have for $y>0$,

$$
\begin{aligned}
& \left|\frac{1}{k_{n}} \mathbf{E}\left(N_{>\left[b\left(n / k_{n}\right) y\right]}(n)\right)-y^{-(2+\delta)}\right| \\
& \quad \leq \frac{1}{k_{n}}\left|\mathbf{E}\left(N_{>\left[b\left(n / k_{n}\right) y\right]}(n)\right)-n p_{>\left[b\left(n / k_{n}\right) y\right]}\right|+\left|\frac{n}{k_{n}} p_{>\left[b\left(n / k_{n}\right) y\right]}-y^{-(2+\delta)}\right| .
\end{aligned}
$$

According to (5.3), the first term is bounded above by $C^{\prime} / k_{n} \rightarrow 0$ as $n \rightarrow \infty$. The second term also goes to 0 by (5.15) as $n \rightarrow \infty$. Hence, as $n \rightarrow \infty$,

$$
\frac{1}{k_{n}} \mathbf{E}\left(N_{>\left[b\left(n / k_{n}\right) y\right]}(n)\right) \rightarrow y^{-(2+\delta)} .
$$

Let $U(t):=\mathbf{E}\left(N_{>[t]}(n)\right)$ and 5.19 becomes: for $y>0$,

$$
\frac{1}{k_{n}} U\left(b\left(n / k_{n}\right) y\right) \rightarrow y^{-(2+\delta)}, \quad \text { as } n \rightarrow \infty .
$$

Since $U(\cdot)$ is a non-increasing function, $U \in R V_{-(2+\delta)}$ by Resnick (2007, Proposition 2.3(ii)). Therefore, Karamata's theorem gives

$$
A \leq \frac{1}{\varepsilon} \int_{M(1-\eta)}^{\infty} \frac{1}{k_{n}} \mathbf{E}\left(N_{>\left[b\left(n / k_{n}\right) y\right]}(n)\right) \frac{\mathrm{d} y}{y} \sim C(\delta, \eta) M^{-(2+\delta)},
$$

with some positive constant $C(\delta, \eta)>0$. Also, $M^{-(2+\delta)} \rightarrow 0$ as $M \rightarrow \infty$, and (5.18) follows. 


\section{Simulation Studies.}

As noted in Remark 9, we fail to prove the consistency of the Hill estimator in Model B using the techniques of Section 5 . In this section, however, we give some simulation results to see how consistent the Hill estimator is in Model B.

The main problem is to choose a proper $k_{n}$. We adopt the threshold selection method proposed in Clauset et al. (2009), which is also widely used in online data sources like KONECT Kunegis (2013). This method is encoded in the plfit script, which can be found at http://tuvalu.santafe.edu/ aaronc/powerlaws/plfit.r). Here is a summary of this method that we refer to it as the "minimum distance method". Given a sample of $n$ iid observations, $Z_{1}, \ldots, Z_{n}$ from a power law distribution with tail index $\alpha$, the minimum distance method suggests using the thresholded data consisting of the $k$ upper-order statistics, $Z_{(1)} \geq \ldots \geq Z_{(k)}$, for estimating $\alpha$. The tail index is estimated by

$$
\hat{\alpha}(k):=\left(\frac{1}{k} \sum_{i=1}^{k} \log \frac{Z_{(i)}}{Z_{(k+1)}}\right)^{-1}, \quad k \geq 1 .
$$

To select $k$, we first compute the Kolmogorov-Smirnov (KS) distance between the empirical tail distribution of the upper $k$ observations and the power-law tail with index $\hat{\alpha}(k)$ :

$$
d_{k}:=\sup _{y \geq 1}\left|\frac{1}{k} \sum_{i=1}^{n} \epsilon_{Z_{i} / Z_{(k+1)}}(y, \infty]-y^{-\hat{\alpha}(k)}\right|, \quad 1 \leq k \leq n .
$$

Then the optimal $k^{*}$ is the one that minimizes the KS distance, i.e.

$$
k^{*}:=\underset{1 \leq k \leq n}{\operatorname{argmin}} d_{k},
$$

and we estimate the tail index and threshold by $\hat{\alpha}\left(k^{*}\right)$ and $Z_{\left(k^{*}+1\right)}$ respectively. This estimator performs well if the thresholded portion comes from a Pareto tail and also seems effective in a variety of non-iid scenarios.

We chose $\delta=-0.5,0,0.5,1,2$ then the theoretical tail indices of degree distributions from Model B were equal to $\alpha:=2+\delta=1.5,2,2.5,3,4$, respectively. For each value of $\delta$, we also varied the number of edges in the network: $n=5000,10000,50000,100000$. For each combination of $(\alpha, n)$, we simulated 500 independent replications of the preferential attachment network using software discussed in Wan et al. (2017) and linked to http://www.orie.cornell. edu/orie/research/groups/multheavytail/software.cfm. For each replication we computed $\hat{\alpha}\left(k^{*}\right)$ using the minimum distance method. We recorded the mean of those 500 estimates in the corresponding entry of Table 1 , based on the combination of $(\alpha, n)$.

We see that when $\delta=-0.5<0$, i.e. $\alpha=1.5$, the minimum distance estimate $\hat{\alpha}\left(k^{*}\right)$ consistently underestimates the tail index, even if the number 


\begin{tabular}{l|cccc}
\hline & \multicolumn{4}{|c}{ Number of Edges } \\
& 5000 & 10000 & 50000 & 100000 \\
\hline$\alpha=1.5$ & 1.481 & 1.484 & 1.484 & 1.488 \\
$\alpha=2$ & 2.061 & 2.028 & 1.998 & 1.990 \\
$\alpha=2.5$ & 2.602 & 2.557 & 2.507 & 2.494 \\
$\alpha=3$ & 3.135 & 3.079 & 3.045 & 2.983 \\
$\alpha=4$ & 3.957 & 3.930 & 3.942 & 3.932 \\
\hline
\end{tabular}

Table 1 Mean values of $\hat{\alpha}\left(k^{*}\right)$ over 500 estimates using the minimum distance method, for each combination of $(\alpha, n)$.

of edges in the network has been increased to $10^{5}$. For the cases where $\delta \geq 0$ (i.e. $\alpha \geq 2$ ), the tail estimates have smaller biases as $n$ increases, as long as the tails are not too "light". When $\alpha=4$, the tail becomes much lighter. Because of the finite sample bias that may occur while applying the minimum distance method to lighter-tailed power laws, increasing the number of edges in the network does not significantly improve the bias of estimates.
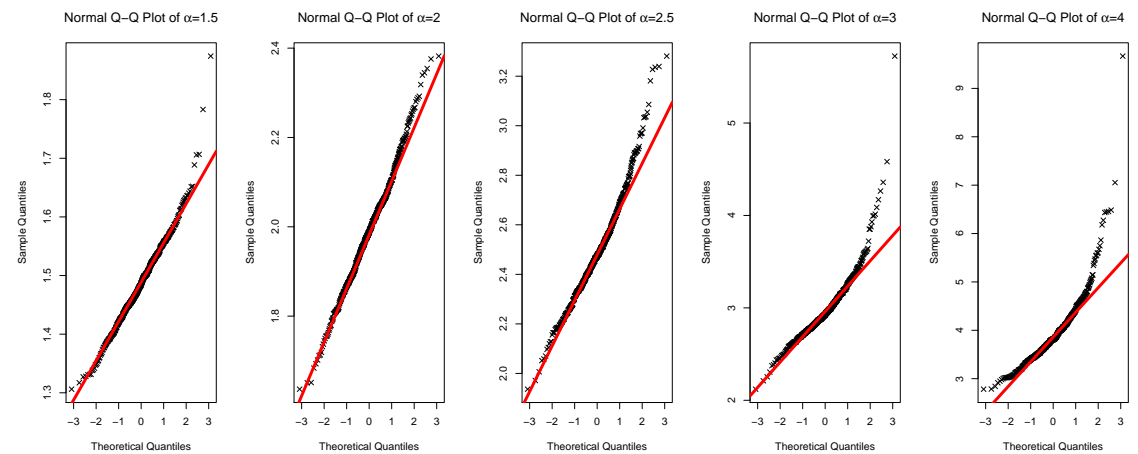

Fig. 6.1 QQ plots of $\hat{\alpha}\left(k^{*}\right)$ with $n=10^{5}$ and $\alpha=1.5,2,2.5,3,4$. The fitted lines in red are the traditional qq-lines used to check normality of the estimates.

In Figure 6.1, we provide the QQ plots of those 500 minimum distance tail estimates $\hat{\alpha}\left(k^{*}\right)$ while holding $n=10^{5}$ and varying $\alpha$ as specified in Table 1 . The fitted lines in red are the traditional qq-lines used to check normality of the estimates. When $\delta \leq 0$ (i.e. the cases where $\alpha=1.5,2)$, QQ plots are consistent with normality of $\hat{\alpha}\left(k^{*}\right)$. However, as $\delta$ increases $(\alpha=2.5,3,4)$, significant departures from the normal distribution are observed and asymptotic normality is not proven theoretically or empirically.

In conclusion, for Model B, simulation results suggest that the Hill estimator is consistent when $\delta \geq 0$ (i.e. the tail index $\alpha \geq 2$ ), but the asymptotic normality is not guaranteed. Since we only have QQ plots of the minimum distance estimates in Figure 6.1, it is still not clear whether this non-normality is due to the minimum distance method or the dependence in the network data. 
We intend to analyze further the consistency for Model B and other variants, as well as the asymptotic behavior of the Hill estimator.

\section{References}

K. B. Athreya. Preferential attachment random graphs with general weight function. Internet Mathematics, 4(4):401-418, 2007. doi: 10.1080/ 15427951.2007.10129150. URL http://dx.doi.org/10.1080/15427951. 2007.10129150

K.B. Athreya, A.P. Ghosh, and S. Sethuraman. Growth of preferential attachment random graphs via continuous-time branching processes. Proceedings Mathematical Sciences, 118(3):473494, August 2008.

S. Bhamidi. Universal techniques to analyze preferential attachment trees: Global and local analysis. available: http://www. unc. edu/ bhamidi/ preferent. pdf, 2007. URL http://www.unc.edu/ bhamidi/preferent. pdf. Preprint.

B. Bollobás, C. Borgs, J. Chayes, and O. Riordan. Directed scale-free graphs. In Proceedings of the Fourteenth Annual ACM-SIAM Symposium on Discrete Algorithms (Baltimore, 2003), pages 132-139, New York, 2003. ACM.

A. Clauset, C.R. Shalizi, and M.E.J. Newman. Power-law distributions in empirical data. SIAM Rev., 51(4):661-703, 2009. ISSN 0036-1445. doi: 10.1137/070710111. URL http://dx.doi.org/10.1137/070710111.

S. Csörgö, E. Haeusler, and D.M. Mason. The quantile-transform-empiricalprocess approach to limit theorems for sums of order statistics. In Sums, Trimmed Sums and Extremes, volume 23 of Progr. Probab., pages 215-267. Birkhäuser Boston, Boston, MA, 1991a.

L. de Haan and S.I. Resnick. On asymptotic normality of the Hill estimator. Stochastic Models, 14:849-867, 1998.

R.T. Durrett. Random Graph Dynamics. Cambridge Series in Statistical and Probabilistic Mathematics. Cambridge University Press, Cambridge, 2010. ISBN 978-0-521-15016-3.

P. Hall. On some simple estimates of an exponent of regular variation. J. Roy. Statist. Soc. Ser. B, 44(1):37-42, 1982. ISSN 0035-9246.

B.M. Hill. A simple general approach to inference about the tail of a distribution. Ann. Statist., 3:1163-1174, 1975.

T. Hsing. On tail estimation using dependent data. Ann. Statist., 19:15471569, 1991.

O. Kallenberg. Random Measures, Theory and Applications. Springer Series in Probability Theory and Stochastic Modelling. Springer eBooks, 2017. ISBN:978-3-319-41598-7.

David G. Kendall. Branching processes since 1873. J. London Math. Soc., 41: 385-406, 1966. ISSN 0024-6107. URL https://doi.org/10.1112/jlms/ s1-41.1.385. 
P. Krapivsky, G. Rodgers, and S. Redner. Degree distributions of growing networks. Phys. Rev. Lett, 86, 2001. doi: 10.1103/PhysRevLett.86.5401. URL http://dx.doi .org/10.1103/PhysRevLett .86.5401.

P.L. Krapivsky and S. Redner. Organization of growing random networks. Physical Review E, 63(6):066123:1-14, 2001.

J. Kunegis. Konect: the Koblenz network collection. In Proceedings of the 22nd International Conference on World Wide Web, pages 1343-1350. ACM, 2013.

D. Mason. Laws of large numbers for sums of extreme values. Ann. Probab., 10:754-764, 1982.

D. Mason and T. Turova. Weak convergence of the Hill estimator process. In J. Galambos, J. Lechner, and E. Simiu, editors, Extreme Value Theory and Applications, pages 419-432. Kluwer Academic Publishers, Dordrecht, Holland, 1994.

S.I. Resnick. Extreme Values, Regular Variation and Point Processes. Springer-Verlag, New York, 1987.

S.I. Resnick. Adventures in Stochastic Processes. Birkhäuser, Boston, 1992.

S.I. Resnick. Heavy Tail Phenomena: Probabilistic and Statistical Modeling. Springer Series in Operations Research and Financial Engineering. SpringerVerlag, New York, 2007. ISBN: 0-387-24272-4.

S.I. Resnick and G. Samorodnitsky. Tauberian theory for multivariate regularly varying distributions with application to preferential attachment networks. Extremes, 18(3):349-367, 2015. doi: 10.1007/s10687-015-0216-2.

S.I. Resnick and G. Samorodnitsky. Asymptotic normality of degree counts in a preferential attachment model. Advances in Applied Probability, 48: 283-299, 7 2016. ISSN 1475-6064. doi: 10.1017/apr.2016.56. URL http: //journals.cambridge.org/article_S0001867816000562.

S.I. Resnick and C. Stărică. Consistency of Hill's estimator for dependent data. J. Appl. Probab., 32(1):139-167, 1995. ISSN 0021-9002.

S.I. Resnick and C. Stărică. Tail index estimation for dependent data. Ann. Appl. Probab., 8(4):1156-1183, 1998. ISSN 1050-5164.

H. Rootzén, M.R. Leadbetter, and L. de Haan. Tail and quantile estimation for strongly mixing stationary sequences. Technical Report 292, Center for Stochastic Processes, Department of Statistics, University of North Carolina, Chapel Hill, NC 27599-3260, 1990.

G. Samorodnitsky, S. Resnick, D. Towsley, R. Davis, A. Willis, and P. Wan. Nonstandard regular variation of in-degree and out-degree in the preferential attachment model. Journal of Applied Probability, 53(1):146-161, March 2016. doi: 10.1017/jpr.2015.15.

S. Tavaré. The birth process with immigration, and the genealogical structure of large populations. Journal of Mathematical Biology, 25(2):161-168, 1987.

R. van der Hofstad. Random Graphs and Complex Networks. Vol. 1. Cambridge Series in Statistical and Probabilistic Mathematics. Cambridge University Press, Cambridge, 2017. ISBN 978-1-107-17287-6. doi: 10.1017/ 9781316779422. URL http://dx.doi.org/10.1017/9781316779422. 
P. Wan, T. Wang, R. A. Davis, and S. I. Resnick. Fitting the linear preferential attachment model. Electron. J. Statist., 11(2):3738-3780, 2017. ISSN 19357524. doi: 10.1214/17-EJS1327.

T. Wang and S. I. Resnick. Asymptotic normality of in- and out-degree counts in a preferential attachment model. Stochastic Models, 33(2):229-255, 2017. doi: 10.1080/15326349.2016.1256219. URL http://dx.doi.org/10.1080/ 15326349.2016.1256219.

T. Wang and S.I. Resnick. Multivariate regular variation of discrete mass functions with applications to preferential attachment networks. Methodology and Computing in Applied Probability, pages 1-14, 2016. ISSN 15737713. doi: 10.1007/s11009-016-9503-x. URL http://dx.doi.org/10.1007/ s11009-016-9503-x.

W. A. O'N. Waugh. Transformation of a birth process into a Poisson process. J. Roy. Statist. Soc. Ser. B, 32:418-431, 1970. ISSN 00359246. URL http://links.jstor.org/sici?sici=0035-9246(1970)32: 3<418: TOABPI>2.0.C0;2-A\&origin=MSN, 